\title{
Prevalence of Campylobacter species in human, animal and food of animal origin and their antimicrobial susceptibility in Ethiopia: a systematic review and meta-analysis
}

\author{
Tizazu Zenebe ${ }^{1 *}$, Niguse Zegeye ${ }^{2}$ and Tadesse Eguale ${ }^{3}$
}

\begin{abstract}
Background: Campylobacter jejuni and Campylobacter coli accounts for most cases of human gastrointestinal infections. The infection occurs through ingestion of contaminated food or water, and direct contact with feces of infected animal or human. Regardless of few local reports of Campylobacter and its antimicrobial susceptibility profile, there is no comprehensive data that show the burden of Campylobacter infection at national level in Ethiopia. This systemic review and meta-analysis aimed to determine the pooled prevalence of Campylobacter and its resistance patterns in Ethiopia from different sources.

Method: A comprehensive literature search of PubMed, Google scholar, Science direct and Google engine search was conducted for studies published from 2000 to July 30,2020 on prevalence and antimicrobial susceptibility of Campylobacter in human, animal and food. The study was conducted according to the Preferred Reporting Items for Systematic Reviews and Meta-Analysis (PRISMA) Checklist. Data from articles was extracted using a standardized data extraction format. The quality of the studies was assessed based on the Newcastle-Ottawa scale. The Q test and $\mathrm{I}^{2}$ test statistic were used to test heterogeneity across studies. The Pooled estimate of prevalence of Campylobacter species and its antimicrobial susceptibility profile was computed by a random effects model using STATA 16.0 software. Results were presented in forest plot, tables, funnel plot and figures with 95\% confidence interval.

Results: A total of 291 articles were retrieved initially. The pooled prevalence of Campylobacter species from different sources was $10.2 \%(95 \% \mathrm{Cl} 3.79,16.51)$. In this meta-analysis, the lowest prevalence was $6.0 \%$ whereas the highest prevalence was $72.7 \%$. In the sub-group analysis, the pooled prevalence was similar in Amhara and Oromia region, higher in Gambella and lower in Sidama. Prevalence of Campylobacter was higher in animals (14.6\%) compared to humans (9\%). The pooled antimicrobial resistance rates of Campylobacter species to different antimicrobials ranged from $2.9-100 \%$. Overall, higher rate of resistance was to cephalothin (67.2\%), gentamicin (67.2\%), and trimethoprimsulfamethoxazole (33.3\%) in Campylobacter isolates from all sources. In isolates from human, resistance to cephalothin was $83 \%$ followed by amoxicillin (80\%), amoxicillin-clavulnate (36\%), trimethoprim-sulfamethpxazole (32\%), clindamycin (31\%) and ceftriaxone (28\%). On the other hand, higher rate of resistance to penicillin (100\%), cephalothin (60\%), ciprofloxacin (71.2\%), and trimethoprim-sulfamethoxazole (39\%) was recorded in isolates from animals.
\end{abstract}

*Correspondence: tizazuzenebe@yahoo.com

1 Department of Medicine, Medical Microbiology Unit, Debre Berhan

University, Debre Berhan, Ethiopia

Full list of author information is available at the end of the article

(c) The Author(s) 2020. This article is licensed under a Creative Commons Attribution 4.0 International License, which permits use, sharing, adaptation, distribution and reproduction in any medium or format, as long as you give appropriate credit to the original author(s) and the source, provide a link to the Creative Commons licence, and indicate if changes were made. The images or other third party material in this article are included in the article's Creative Commons licence, unless indicated otherwise in a credit line to the material. If material is not included in the article's Creative Commons licence and your intended use is not permitted by statutory regulation or exceeds the permitted use, you will need to obtain permission directly from the copyright holder. To view a copy of this licence, visit http://creativecommons.org/licenses/by/4.0/. The Creative Commons Public Domain Dedication waiver (http://creativecommons.org/publicdomain/zero/1.0/) applies to the data made available in this article, unless otherwise stated in a credit line to the data. 
Conclusion: The present study highlights the burden of Campylobacter species in the country and higher rate of resistance among investigated isolates. Designing appropriate prevention strategies and further local in-depth studies are recommended to establish actual epidemiological burden of the bacteria in the country.

Keywords: Systematic review, Meta-analysis, Prevalence, Antimicrobials Resistance, Campylobacter species, Ethiopia

\section{Introduction}

Different enteric pathogens such as bacteria, virus, and parasites cause gastrointestinal infections in human [1]. Campylobacter species account for most cases of human bacterial gastrointestinal infections worldwide [2]. The genus Campylobacter belongs to the family Campylobacteraceae [3] and the genus Campylobacter contains 39 species with 16 subspecies [4]. Campylobacter jejuni and C. coli are commonly responsible to gastroenteritis in humans [3]. Campylobacter is one of the most frequently isolated bacteria from stools of infants with diarrhoea in developing countries, mainly due to contaminated food or water [5]. Campylobacteriosis is a common cause of human gastroenteritis in developing and industrialized countries [2]. Campylobacter jejuni, and $C$. coli are zoonotic pathogens where poultry, wild birds, cattle, sheep, and pigs are known source of infection $[3,6]$. Poultry is a major reservoir and source of transmission of campylobacteriosis to humans [7]. Ingestion of contaminated food or water (improperly cooked poultry, untreated water, and unpasteurized milk), and direct contact with fecal material from infected animals or people are the major means of transmission of $C$ jejuni and $C$ coli [6]. Campylobacter species have well-known virulence and survival mechanisms to cause disease in human and animal [8]. In East Africa, Campylobacter infections have been recorded in both rural and urban areas, particularly among children and the prevalence varies between countries [9]. The spectrums of disease caused by Campylobacter include acute enteritis (gastroenteritis), extraintestinal infections (e.g. bacteremia, abscess, meningitis) and postinfectious complications (e.g. Guillain-Barre syndrome, reactive arthritis, and irritable bowel syndrome) $[6,7]$.

Most of the time, Campylobacter infection is a selflimiting and requires no therapeutic intervention other than supportive therapy. However, antimicrobials are employed in patients with severe, persistent, and extra intestinal campylobacteriosis and in immune-compromised patients [7]. The use of antimicrobials in agriculture led to a dramatic increase in antimicrobials resistance in several human pathogens originating from animals, including Campylobacter species [7].

In Ethiopia, only a few studies have been conducted on occurrence of Campylobacter in human, animal and food [10-14]. However, some of these studies reported high prevalence of Campylobacter with and reported various risk factors and antimicrobial resistance profile $(13,15)$. An emerging poultry production has been reported as a major contributing factor to environmental exposure to Campylobacter in Ethiopia (16). Plenty of reservoirs of Campylobacter create risk for human infection through contaminations with animal, food and environments (3, $6,17)$. There is no comprehensive data that shows the burden of Campylobacter at national and regional level. The aim of this review was to generate comprehensive evidences on prevalence and antimicrobial susceptibility profile of Campylobacter in human, animal and food in Ethiopia. The findings of this study are expected to provide data that can be employed for prevention and control of Campylobacter infections as well as to provide gaps for further research in the country.

\section{Methods}

Study design

A systematic review and meta-analysis was conducted to estimate the prevalence and antimicrobials resistance patterns of Campylobacter in Ethiopia. The study was conducted according to the Preferred Reporting Items for Systematic Reviews and Meta-Analysis (PRISMA) Checklist [18].

\section{Literature search strategies for relevant studies}

A comprehensive search of literatures published from 2000 to July 30, 2020 was performed in the following databases: PubMed, Google Scholar, Science Direct, Google, and manually by obtaining hard copy of locally published articles directly from authors and local libraries. Campylobacter, antibacterial agents, Antibiotics, Resistance, Susceptibility, and Ethiopia were used as search arms. We used search terms using Boolean operators for PubMed, Google Scholar and Science Direct: ((Campylobacter) AND (Antimicrobial OR Antibiotics)) AND (Resistance OR Susceptibility)) AND (Ethiopia).

\section{Study eligibility criteria}

All available studies and data were incorporated based on the following predefined eligibility criteria. Studies conducted in Ethiopia, published articles, cross-sectional study, and articles reported in English language were used as inclusion criteria. The exclusion criteria were 
articles which were with duplicate or overlapping data, and articles without full text available.

\section{Study selection}

Records identified from various sources with the search strategies were exported to Endnote reference manager software version 7. Duplicate records were identified, recorded and removed. For this, two authors independently screened the title and abstracts with the predefined inclusion criteria. Two authors were also independently assigned to collect full texts and evaluate their eligibility for final inclusion. When discrepancies between two authors occurred, the third author played a role in resolving the issue through discussion and consensus.

\section{Measurement of outcome variables}

The outcomes of the study were prevalence of Campylobacter and antimicrobial resistance pattern. The prevalence was calculated by dividing the numbers of samples positive for Campylobacter by the total number of tested samples.

\section{Data extraction}

All necessary data from included articles was extracted using a standardized data extraction format by two authors independently. Any disagreement during the data extraction was resolved through discussion and consensus. The primary author of the original research was contacted for additional information or to clarify method details as needed. The data extraction format for prevalence of Campylobacter included primary author, publication year, region, sample size, sample source, study population, diagnostic method, prevalence, species, and quality score. In addition, number of isolates, antimicrobials tested and percentage of resistance were also extracted.

\section{Quality assessment}

Two authors independently assessed the risk of bias for each original study. The quality of study was evaluated according to Newcastle-Ottawa scale adapted for cross sectional studies [19] and graded out of 10 points (10 stars). The tool has three major sections: methodological quality (with 5 stars), comparability of the study (with 2 stars) and outcomes related to statistical analysis (with 3 stars). The mean score of two authors took for final decision, and studies with score greater than or equal to five were included for systemic review and meta-analysis (Additional file 1: Table S2).

\section{Data processing and analysis}

The relevant extracted data was recorded using format prepared in Microsoft Excel. The data analysis was done using STATA 16.0 software. The data prepared in Microsoft excel were imported into the STATA for outcome measures and subgroup analyses. In the determination of variation in true effect sizes across population (clinical heterogeneity), restricted maximum-likehood random effect model was applied. The original articles were described using forest plot, funnel plot and tables. Random effect model was used to compute the pooled prevalence of Campylobacter and resistance rate. The estimated pooled prevalence with $95 \%$ confidence interval by forest plot and publication bias by funnel plot was presented. Sub group analysis was performed based on publication year, region, and study population.

Heterogeneity among reported prevalence was assessed by computing p-values of Cochrane Q-test and $I^{2}$ statistics [20]. Cochrane Q-test evaluates the existence of heterogeneity and $\mathrm{p}<0.1$ was considered as statistically significant [21]. The $I^{2}$ statistics provides an estimate of the percentage of the variability in effect estimates that is due to heterogeneity rather than sampling error or chance differences. $\mathrm{I}^{2}$ values of $25 \%, 50 \%$ and $75 \%$ are considered to represent low, medium and high heterogeneity respectively [20, 21]. Begg's rank test and Egger's regression test are among various statistical tests used for checking publication bias in the funnel plot [22]. Begg's rank test was used to examine the correlation between the effect sizes and their corresponding sampling variances and a strong correlation implies publication bias. Egger's test regresses the standardized effect sizes on their precisions; in the absence of publication bias, the regression intercept is expected to be zero [22]. Begg's rank test and Egger's tests at $5 \%$ significant level were used to check publication bias [22, 23].

\section{Results}

A total of 291 articles were initially retrieved for the prevalence of Campylobacter species and antimicrobial susceptibility from different sources in Ethiopia using different data bases described above. As a result of duplication, 48 articles were removed. After further screening, 160 articles which were books, review articles, systemic reviews, book chapters and encyclopaedia were excluded. Based on the eligibility criteria, 69 articles were excluded and 14 were selected for their full text articles. Finally, 2 articles were excluded due to insufficient information and the remaining 12 articles were included for systemic review and meta-analysis (Fig. 1). 


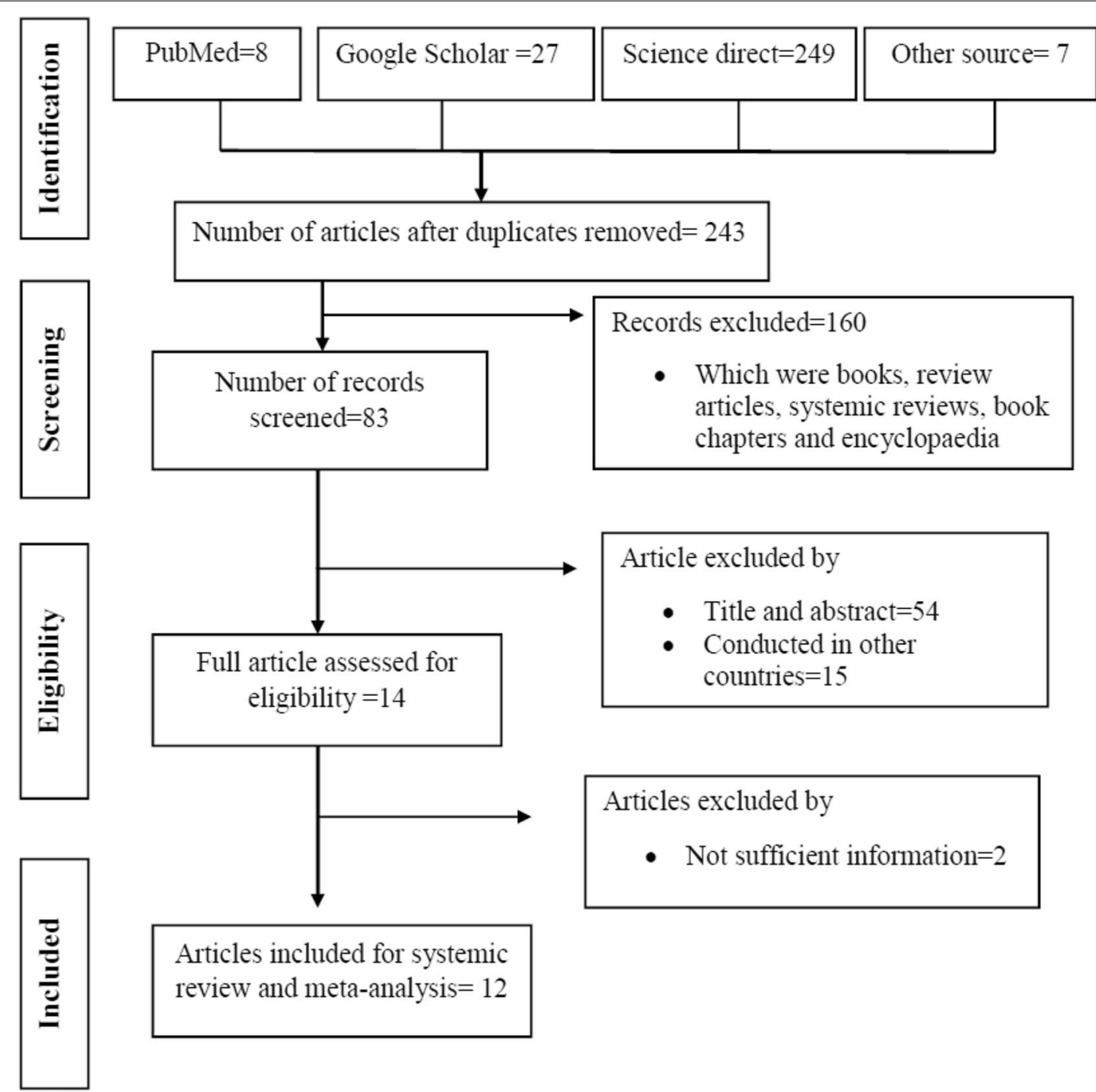

Fig. 1 Flow chart of study selection for systematic review and meta-analysis of the prevalence of Campylobacter species in different parts of Ethiopia

\section{Description of search results}

All the included 12 articles (Table 1) were cross sectional in their study design and published from 2004 to 2020. The total sample size for these selected studies was 4230 (ranging from 158-540. These studies were conducted in four regional states, namely Amhara, Oromia, Sidama, and Gambella regions. Majority of the studies were conducted in Oromia [10, 15, 24-26] and Amhara [11, 13, $27,28]$ region. The studies involved humans, animals and food of animal origin; more than $90 \%$ were conducted in humans and animals. Among all the studies, one study isolated Campylobacter from both humans and animals which were separately analysed. Data on susceptibility profile of Campylobacter species was included for a total of 14 antimicrobials. The inclusion of antimicrobials in each study ranged from 4-14 antimicrobials, 50\% of the studies tested susceptibility of Campylobacter to 9 or more antimicrobials.

\section{Prevalence of Campylobacter species}

In the present systemic and meta-analysis of 12 included articles, the pooled prevalence of Campylobacter species was $10.2 \%$ (95\% CI $3.79,16.51)$. There was no heterogeneity observed across the included studies $\left(\mathrm{I}^{2} 0.01 \%\right.$; $\mathrm{Q}=3.23, \mathrm{p}=1.00)$. However, random effect model with restricted maximum-likelihood method was used to estimate the pooled prevalence of Campylobacter species from different sources in Ethiopia (Fig. 2). In this meta-analysis, the lowest prevalence was 6.1\% [29] from stool sample of HIV infected individuals and highest 


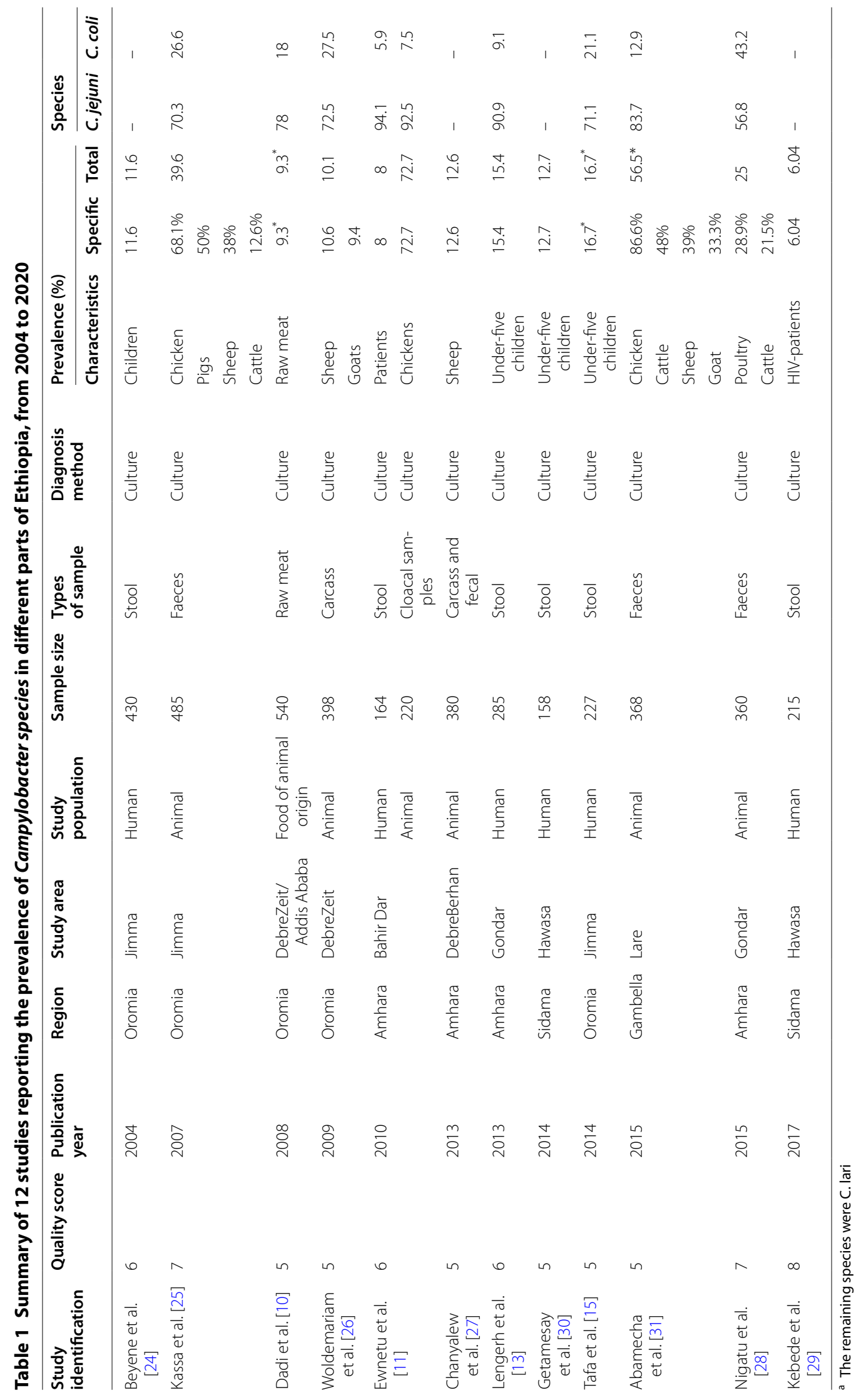




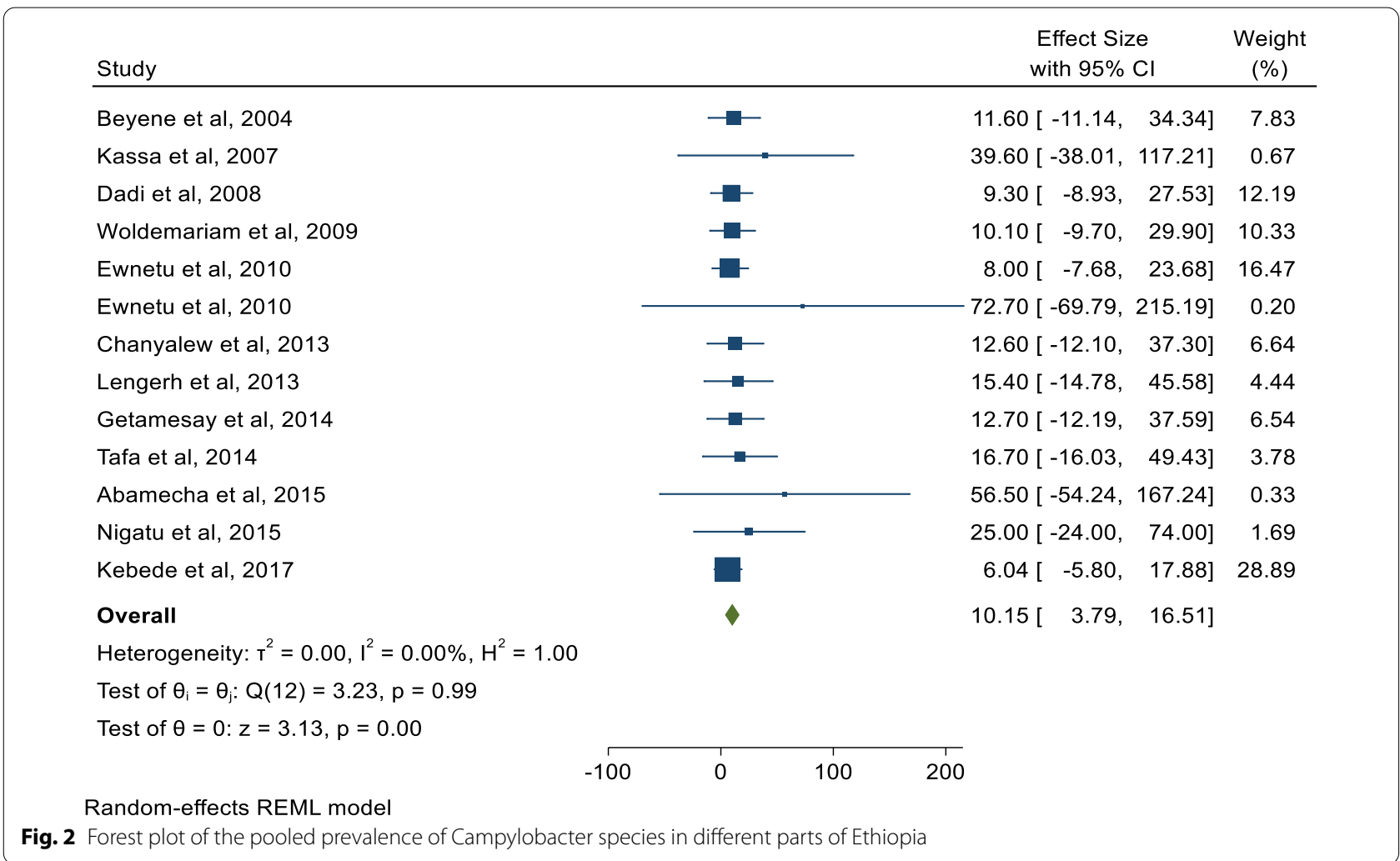

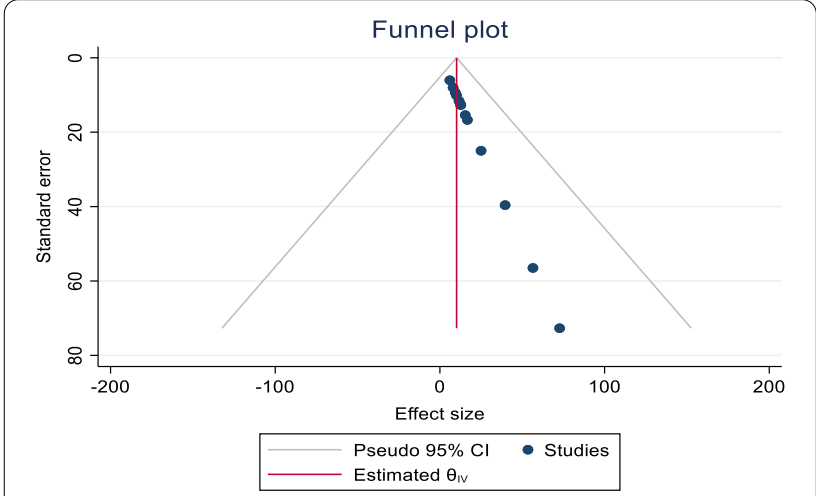

Fig. 3 Funnel plot with $95 \%$ confidence limits of the prevalence of Campylobacter species in different parts of Ethiopia

prevalence was $72.7 \%$ from cloacal samples of chicken [11]. The Campylobacter species including C. jejuni, and C. coli (C. lari in few studies too) were reported at species level in $75 \%$ of the studies. Significantly predominant species with pooled prevalence of $75.0 \%$ (95\% CI 25.36, 124.66) were C. Jejuni compared to C. coli $9.4 \%$ (95\%CI $2.19,16.68)$ in Ethiopia. Funnel plot was used to show the distribution of the studies and showed symmetrical distribution of effect estimate (Fig. 3). The Egger's test showed that there was no statistically significant publication bias in estimating the prevalence of Campylobacter species from different sources $(\mathrm{p}=0.0725)$.

\section{Subgroup analysis}

A subgroup analysis for publication year, region, and study population (human, animal and food of animal origin) was done (Table 2). In most cases, sub group analysis was done to investigate source of heterogeneity (effect difference in relevant subgroups), and to provide effect estimate (prevalence) for clinically relevant subgroups [32]. Regardless of absence of heterogeneity, we performed subgroup analysis to provide effect estimate for clinically relevant subgroups. This was with assumption that the effect estimate between clinically relevant subgroups will vary. In considering the small studies effect, Trim and Fill analysis was used to adjust the final pooled estimate for each subgroup. The estimate pooled prevalence of Campylobacter species from different sources was not changed within the two decades period, from 2000-2010 (10.1, 95\% CI 0.88, 19.31) and 2011-2020 $(10.2,95 \% \mathrm{CI} 1.40,19.00)$. The pooled prevalence in the two regions, Amhara $(11.6,95 \% \mathrm{CI}-0.16,23.29)$ and Oromia $(11.4,95 \%$ CI $0.66,22.23)$ was almost the same. Lowest prevalence was seen in Sidama region $(7.3,95 \%$ CI - 3.27, 17.96) whereas highest prevalence was seen in 
Table 2 Subgroup estimate pooled prevalence of Campylobacter species in animal, human and food in Ethiopia

\begin{tabular}{lllll}
\hline Variables & Characteristics & Included studies & Sample size & Pooled prevalence (95\% Cl) \\
\hline Publication year & $2000-2010$ & 6 & 2237 & $10.09(0.88,19.31)$ \\
\multirow{2}{*}{ Region } & $2011-2020$ & 7 & 1993 & $10.20(1.40,19.00)$ \\
& Amhara & 5 & 1409 & $11.57(-0.16,23.29)$ \\
& Oromia & 5 & 2080 & $11.44(0.66,22.23)$ \\
Study population & Gidama & 2 & 373 & $7.27(-3.27,17.96)$ \\
& Gambella & 1 & 368 & $56.50(-54.24,167.24)$ \\
& Animal & 6 & 2211 & $14.60(0.32,28.88)$ \\
& Human & 6 & 1479 & $9.00(1.28,16.72)$ \\
\end{tabular}

Gambella $(56.5,95 \%$ CI - 54.24, 167.24). Higher prevalence was seen in animals (14.6: 95\%CI 0.32, 28.88) compared to human and food. The prevalence was almost similar in human and food of animal origin.

\section{Antimicrobial susceptibility profile of Campylobacter species}

The percentage of antimicrobial resistance rates of Campylobacter species to different antimicrobials varied from $0-100 \%$ (Table 3). Over $60 \%$ of Campylobacter isolates were resistant to Penicillin, Ampicillin, Amoxicillin, Tetracycline, Cephalothin, Gentamicin, Ciprofloxacin, and Trimethoprim-sulfamethoxazole. Relatively, Streptomycin, Nalidixic acid, Chloramphenicol and Norfloxacin were effective to about $50 \%$ of Campylobacter isolates.

The pooled resistance rates of Campylobacter isolates to14 antimicrobials was found to be varied, ranged from 2.9-100\% (Additional file 1: Table S1). A single study showed $100 \%$ resistance of Campylobacter isolates to penicillin. Campylobacter isolates showed significantly higher resistance rate to Cephalothin (67.2\%), Gentamicin (67.2\%), and Trimethoprim-sulfamethoxazole (33.3\%). Isolates were relatively more sensitive to Amoxicillin, Erythromycin, Streptomycin, Nalidixic acid, Chloramphenicol, and Norfloxacin but not statistically significant $(\mathrm{p}<0.05)$.

A sub group analysis was performed to examine estimate pooled antimicrobial resistance rate to Campylobacter in human, animal and food (Table 4). The Campylobacter isolates from human showed higher resistance against amoxicillin (80\%), cephalothin (83\%), ceftriaxone (28\%), amoxicillin-clavulnate (36\%), trimethoprim-sulfamethpxazole (32\%), and clindamycin (31\%). Campylobacter isolates from animal also showed higher resistance to penicillin (100\%), cephalothin (60\%),

Table 3 Percentage of antibacterial resistance rates of Campylobacter species in Ethiopia, from 2004 to 2020

\begin{tabular}{|c|c|c|c|c|c|c|c|c|c|c|c|c|c|c|c|c|c|c|c|}
\hline \multirow[t]{2}{*}{ Studies } & \multirow{2}{*}{$\begin{array}{l}\text { Number } \\
\text { of isolates }\end{array}$} & \multirow[b]{2}{*}{$\mathbf{P}$} & \multicolumn{12}{|c|}{ Antimicrobials resistance rate reported by 12 studies (\%) } & \multirow[b]{2}{*}{ NOR } & \multirow[b]{2}{*}{ CRO } & \multirow[b]{2}{*}{$\mathbf{T} / \mathrm{S}$} & \multirow[b]{2}{*}{ CD } & \multirow[b]{2}{*}{ K } \\
\hline & & & AMP & AML & $\mathbf{E}$ & STR & $\mathbf{T}$ & NA & CF & GM & CIP & CL & $\mathrm{A} / \mathrm{C}$ & DC & & & & & \\
\hline Lengerh et al. [13] & 44 & - & 68.2 & - & 27.7 & - & 56.8 & 9.1 & 88.9 & 18.2 & 16 & 11.4 & 36.4 & 15.9 & 11.6 & 27.7 & 54.5 & 40.9 & - \\
\hline Beyene et al. [21] & 50 & - & 50 & - & 10 & - & 14 & - & - & 0 & - & 0 & - & - & - & - & 60 & - & 0 \\
\hline Dadi et al. [10] & 50 & - & 10 & 6 & 2 & 20 & 10 & - & - & 14 & - & 4 & - & - & - & - & - & - & 12 \\
\hline Kebede et al. [27] & 13 & - & - & - & 23.1 & - & 23.1 & 7.7 & - & 0 & 7.7 & 53.8 & - & - & 0 & 0 & 61.5 & - & - \\
\hline Getamesay et al. [28] & 20 & - & 30 & 80 & 55 & - & 15 & 20 & 70 & 70 & 10 & 0 & - & - & - & 0 & 20 & - & - \\
\hline Chanyalew et al. [25] & 48 & - & 33.3 & - & - & 4.2 & 20.8 & 2.1 & 100 & - & - & - & - & - & - & - & - & - & - \\
\hline Tafa et al. [24] & 38 & - & 76 & - & 18.4 & - & 39.5 & 10.5 & 100 & 13.2 & 15.8 & 31.6 & - & 23.7 & 10.5 & - & 68.4 & 26.3 & - \\
\hline \multirow[t]{2}{*}{ Ewnetu et al. [11] } & 15 & - & 18.8 & - & 18.8 & 25 & 22.2 & 12.5 & - & - & - & - & - & - & - & - & - & - & - \\
\hline & 160 & - & 34.1 & - & 14.9 & 20.5 & 30.1 & - & - & - & - & - & - & - & - & - & - & - & - \\
\hline Kassa et al. [22] & 192 & - & 19.8 & - & 2.6 & 6.3 & 2.6 & 6.3 & 96.9 & 0.5 & - & - & - & - & 2.2 & - & 38.5 & 2.1 & - \\
\hline Abamecha et al. [29] & 208 & - & 38.9 & - & 50.5 & - & 69.2 & 3.8 & 100 & - & 71.2 & 51.4 & - & - & 54.3 & - & - & 45.7 & - \\
\hline Nigatu et al. [26] & 84 & 100 & 8.3 & - & 60 & 13.1 & 35.7 & 0 & 40.5 & - & - & - & - & - & - & - & - & - & - \\
\hline
\end{tabular}

AMP Ampicillin, AML Amoxicillin, E Erythromycin, STR Streptomycin, TTetracycline, NA Nalidixic acid, P Penicillin, CF Cephalothin, GM Gentamicin, CIP Ciprofloxacin, $C L$ Chloramphenicol, $A$ /CAmoxicillin with clavulanic acid, DC Doxycycline, NOR Norfloxacin, $C R O$ Ceftriaxone, $T / S$ Trimethoprim-sulfamethoxazole, $C D$ Clindamycin, $K$ Kanamycin 
Table 4 Percentage of pooled resistance rates of antimicrobials to Campylobacter isolates from human, animal and food in Ethiopia, from 2004 to 2020

\begin{tabular}{llll}
\hline Antimicrobials & \multicolumn{2}{l}{ Pooled antimicrobial resistance rate $\mathbf{( 9 5 \%} \mathbf{C l})$} & Food \\
\cline { 2 - 4 } & Human & Animal & - \\
\hline Penicillin & - & $100(-96,296)$ & $10(-9.6,29.6)$ \\
Ampicillin & $28.41(-0.09,56.91)$ & $13.14(-0.90,27.18)$ & $6(-5.76,17.76)$ \\
Amoxicillin & $80(-76.8,236.8)$ & - & $2(-1.92,5.92)$ \\
Erythromycin & $15.82(1.7,29.93)$ & $4.01(-3.86,11.89)$ & $20(-19.2,59.2)$ \\
Streptomycin & $25(-24,74)$ & $5.79(-0.74,12.32)$ & $10(-9.6,29.6)$ \\
Tetracycline & $18.7(2.37,35.04)$ & $10.37(-7.03,27.77)$ & - \\
Doxycycline & $18.32(-7.56,44.2)$ & - & - \\
Nalidixic acid & $9.91(0.86,18.96)$ & $2.8(-0.66,6.25)$ & - \\
Cephalothin & $82.52(-11.93,176.97)$ & $60.05(-4.71,124.8)$ & - \\
Ceftriaxone & $27.7(-26.59,81.99)$ & & $14(-13.44,41.44)$ \\
Gentamicin & $16.18(-4.53,36.88)$ & $0.5(-0.48,1.48)$ & - \\
Ciprofloxacin & $10.23(-0.23,20.74)$ & $71.2(-68.35,210.75)$ & $4(-3.84,11.84)$ \\
Chloramphenicol & $15.26(-5.35,35.87)$ & $51.4(-49.34,152.14)$ & - \\
Amoxicillin-clavulanate & $36.4(-34.94,107.74)$ & - & - \\
Norfloxacin & $11(-4.26,26.25)$ & $2.29(-2.02,6.59)$ & - \\
Trimethoprim-sulfamethoxazole & $32.26(-0.44,64.96)$ & $38.5(-36.96,113.96)$ & - \\
Clindamycin & $30.57(-12.79,73.93)$ & $2.19(-1.92,6.30)$ & $-12(-11.52,35.52)$ \\
Kanamycin & - & - &
\end{tabular}

ciprofloxacin (71.2\%), and trimethoprim-sulfamethoxazole (39\%). In both cases the resistance was not statistically significant. Campylobacter isolates from animal and human showed higher resistance to cephalothin and trimethoprim-sulfamethoxazole. The Campylobacter strains isolated from human showed significant resistance to erythromycin (16\%) and tetracycline (19\%).

\section{Discussion}

Human campylobacteriosis is a public health concern globally because of rise in the incidence of Campylobacter infection, and trend of increasing resistance to antimicrobial agents [33]. Thermotolerant Campylobacter species (C jejuni, C coli, C. lari, and C. upsaliensis) have clinical relevance in human [6]. Currently, the most relevant species within the genus is $C$. jejuni, a leading cause of bacterial gastroenteritis in humans followed by $C$. coli (1 to $25 \%$ of all Campylobacter-related diarrheal diseases) [34]. Campylobacter jejuni is also the most significant pathogenic Campylobacter in animals followed with other species such as C. fetus [35]. However, today there are other emerging Campylobacter species that cause disease in human and animals [34]. Most of the studies done in Ethiopia included only C. jejuni, and C. coli. Majority of studies used in the present systematic review and meta-analysis reported only C. jejuni and C. coli in different sources (human, animal and food).
The pooled prevalence of Campylobacter species in the present systemic review and meta-analysis was $10.2 \%$ with $C$. jejuni as predominant species. Systemic review conducted on studies conducted in Sub-Saharan Africa showed that prevalence of Campylobacter species ranged from $1.7 \%-62.7 \%$ in humans and $1.2 \%-80 \%$ in animals [36]. Ethiopia as part of Sub-Saharan Africa country shares the burden of Campylobacter infection. In the sub-group analysis, during the two decades, there was no change in prevalence of Campylobacter. This is presumably due to the fact that there was no specific intervention strategy conducted to prevent and control Campylobacter. Studies from other parts of the world showed increasing incidence and prevalence of Campylobacter species as emerging infections [34] which need serious attention against burden of Campylobacter infection.

The pooled prevalence in Amhara region (11.6\%) and Oromia (11.4\%) was almost the same. This similarity may be due to communality of contributing factors such as day to day activities, agricultural practice, and life style of the communities who live in these regions which could have contributed to the transmission of Campylobacter infection in similar manner. However, one of the studies conducted in Amhara region reported relatively higher prevalence of Campylobacter (72.7\%) compared to the other reports in both regions. This may be due to difference in study population, for example this study was 
conducted in chickens. Chickens appear to be important hosts for Campylobacter compared to the other species [4] and suggests the importance of chicken in the transmission of Campylobacter in the area. In majority of the studies included in the present systemic review and meta-analysis, higher prevalence of Campylobacter was reported from Chicken. Lower prevalence was reported in study from Sidama region (7.3\%) whereas higher prevalence was reported from Gambella (56.5\%). This may be due to few numbers of studies done in these regions (report bias) or actual difference. Hence, there is a need for more research in the area.

Higher prevalence was reported in animals (14.6\%) compared to human and food. And this may be due to the fact that most of domestic animals can serve as reservoir for Campylobacter [4]. A systematic review and meta-analysis of Campylobacter from food animals and meat in Africa reported 37.7\% prevalence estimate of Campylobacter [37]. Gahamanyi et al. [36] also reported prevalence of Campylobacter in animal ranged from $1.2 \%-80 \%$. The higher prevalence in the present study therefore agreed with studies conducted elsewhere and could suggest the importance of Campylobacter related to animals in Ethiopia. In the sub-group analysis, the pooled prevalence of Campylobacter species in human was $9 \%$ which is lower compared to reports by Gahamanyi et al. [36], 62.7\% in Nigeria, 21\% in Malawi, and 20.3\% in South Africa. The difference may be due to difference in detection methods used by different studies. A recent study done in Eastern Ethiopia reported 50\% using PCR and $88 \%$ using meta-total RNA sequencing from stool of children [38]. The mean prevalence of Campylobacter in Sub-Saharan Africa was $18.6 \%$ for all age groups and 9.4\% for under-five children [36]. This is in line with the present estimation, particularly for under-five children. The study population in majority of the present study for human were children or under-five children (66.7\%). This suggests that the burden of Campylobacter in underfive children in Ethiopia is similar to other Sub-Saharan Africa countries. Campylobacter is well recognized as the leading cause of bacterial foodborne diarrheal diseases [39]. The 9.3\% prevalence of Campylobacter in a single study in raw meat agrees with Pallavi et al. [40] who reported $17.3 \%$ in chicken meat.

Resistance rates of Campylobacter species to different antimicrobials varied from $0-100 \%$ in the present study. This agrees with Gahamanyi et al. [36] who reported resistance to commonly used antimicrobials ranging from $0-100 \%$. The pooled rate of resistance of Campylobacter isolates to different antimicrobials was erythromycin (3.1\%), ampicillin (14.3\%), tetracycline (17.1\%), nalidixic acid (3.7\%), and ciprofloxacin (10.6\%) in the present study. Contrary to this, Hlashwayo et al. [4] reported higher resistance rate to erythromycin (44\%), ampicillin (39\%), tetracycline (33\%), nalidixic acid (31\%) and ciprofloxacin (30\%). This discrepancy may be due to difference in study population, the present study include human, animal and food whereas the previous study was based only on animal isolates. In the present study Campylobacter species showed significantly higher resistance rate to cephalothin (67.2\%), gentamicin (67.2\%), and trimethoprim-sulfamethoxazole (33.3\%). Trends in fluoroquinolone-resistance in samples from animal and human showed increased resistance among isolates of members of the genus Campylobacter [41]. Importantly, based on the present systemic and meta-analysis, antimicrobials with good activity against Campylobacter species included amoxicillin, erythromycin, streptomycin, nalidixic acid, chloramphenicol, and norfloxacin.

The present finding highlights the frequent occurrence of resistance strains of Campylobacter to commonly used antimicrobials in human, animal and food in Ethiopia (Table 4). The Campylobacter isolated from human and animal showed higher resistance against commonly used antimicrobials. The finding agrees with Hlashwayo et al. [4] reports in which most Campylobacter isolates were resistant to erythromycin (44\%), ampicillin (39\%), tetracycline (33\%), nalidixic acid (31\%) and ciprofloxacin (30\%). The Campylobacter isolated from human showed significant resistance against erythromycin $(16 \%)$ and tetracycline (19\%). Antimicrobial use in agricultural industry considered as a root cause of antimicrobials resistance in Campylobacter and other foodborne pathogens [42]. In addition misuse of antimicrobials in poultry industry is found to be source for antimicrobial resistant bacterial isolates in Ethiopia [43]. This could be due to irrational use of antimicrobials in food production in Ethiopia [44].

Majority of the studies were done by culture only which is a challenge for the growth of Campylobacter for reporting actual prevalence. The other limitations include less number of studies done in Ethiopia, absence of data from other regions and few number of articles were included for this systemic and meta-analysis. Few studies were available in food, animals and human that limited comparisons of the prevalence and resistance profile.

\section{Conclusion}

The pooled prevalence of Campylobacter species was 10.2\% with higher prevalence in animal and predominant species was $C$. jejuni. The prevalence of Campylobacter species varied among region. The study found different levels of occurrence of resistant Campylobacter strains in human, animal and food in Ethiopia. Campylobacter isolated either from human or animal or foods were showed higher resistance against amoxicillin, cephalothin, ceftriaxone, amoxicillin-clavulnate, clindamycin, penicillin, ciprofloxacin, and 
trimethoprim-sulfamethoxazole. Majority of the studies were done in Amhara and Oromia region, and the design did not include other emerging species.

The present study highlights the burden of Campylobacter species and showed gap of data on Campylobacter in many other regions of the country. Infection with resistant strains of Campylobacter could challenge the management of the infection unless the right prevention strategies are design. Further studies involving advanced techniques and targeting other emerging Campylobacter species are recommended to understand full picture of the burden Campylobacter in the country. Furthermore, studies focusing on the transmission dynamics of resistance strains of Campylobacter between human, animal and food is recommended.

\section{Supplementary Information}

The online version contains supplementary material available at https://doi. org/10.1186/s12941-020-00405-8.

Additional file 1: Table S1. Percentage of pooled antimicrobial resistance rates of 14 antimicrobials to Campylobacter isolates in Ethiopia, from 2004 to 2020. Table S2. Critical appraisal of studies.

\section{Acknowledgements}

We don't have any person or organization to acknowledge.

\section{Authors' contributions}

TZ was the principal investigator who contributed to origin, the idea and design of the study. TZ, TE and NZ collected, entered, analysed, interpreted the data, prepared the manuscript, contributed to data analysis, interpretation and drafted the manuscript. All authors read and approved the final manuscript.

\section{Funding}

There was no any funding or sponsoring organization for this paper.

\section{Availability of data and materials}

There is no remaining data and materials; all information is clearly presented in the main manuscript.

\section{Ethics approval and consent to participate}

Not applicable.

\section{Consent for publication \\ Not applicable.}

\section{Competing interests}

The authors declare that they have no competing interests.

\section{Author details}

${ }^{1}$ Department of Medicine, Medical Microbiology Unit, Debre Berhan University, Debre Berhan, Ethiopia. ${ }^{2}$ Department of Medical Laboratory Science, Debre Berhan University, Debre Berhan, Ethiopia. ${ }^{3}$ Aklilu Lemma Institute of Pathobiology, Addis Ababa University, Addis Ababa, Ethiopia.

Received: 24 August 2020 Accepted: 30 November 2020

Published online: 10 December 2020

\section{References}

1. Hodges K, Gill R. infectious diarrhea: cellular and molecular mechanisms. Gut Microbes. 2010;1(1):4-21.
2. Mughal MH: Campylobacteriosis: A Global Threat. Biomed J Sci \& Tech Res 2018, 11(5).

3. Facciolà A, Riso R, Avventuroso E, Visalli G, Delia SA, Laganà P. Campylobacter: from microbiology to prevention. J Prev Med Hyg. 2017:58:E79-92.

4. Hlashwayo DF, Sigaúque B, Bila CG: Epidemiology and antimicrobial resistance of Campylobacter spp. in animals in Sub-Saharan Africa: A systematic review. Heliyon 2020, 6:e03537.

5. Coker AO, Isokpehi RD, Thomas BN, Amisu KO, Obi CL. Human Campylobacteriosis in Developing Countries. Emerg Infect Dis. 2002;8(3):237-43.

6. Fitzgerald C. Campylobacter. Clin Lab Med. 2015;35(2):289-98.

7. Kaakoush NO, Castaño-Rodríguez N, Mitchell HM, Man SM: Global Epidemiology of Campylobacter Infection. Clin Microbiol Rev 22015, 28(3):687-720.

8. Bolton DJ. Campylobacter virulence and survival factors. Food Microbiol. 2015;48:99e108.

9. Asuming-Bediako N. Kunadu AP-H, Abraham S, Habib I: Campylobacter at the Human-Food Interface: The African Perspective. Pathogen. 2019:8(87):1-30.

10. Dadi L, Asrat D. Prevalence and antimicrobial susceptibility profiles of thermotolerant Campylobacter strains in retail raw meat products in Ethiopia. Ethiop J Health Dev. 2008;22(2):195-200.

11. Ewnetu D, Mihret A. Prevalence and Antimicrobial Resistance of Campylobacter Isolates from Humans and Chickens in Bahir Dar Ethiopia. Foodborne Pathog. Dis. 2010;7(6):667-70.

12. Kassa T, Gebre-selassie S, Asrat D. The prevalence of thermotolerant Campylobacter species in food animals in Jimma Zone, southwest Ethiopia. Ethiop J Health Dev. 2005;19(3):225-9.

13. Lengerh A, Moges F, Unakal C, Anagaw B: Prevalence, associated risk factors and antimicrobial susceptibility pattern of Campylobacter species among under five diarrheic children at Gondar University Hospital, Northwest Ethiopia. BMC Pediatrics 2013, 13(82).

14. Mitike G, Kassu A, Genetu A, Nigussie D: Campylobacter Enteritis among Children in Dembia District, Northwest Ethiopia. EAM 2000:654-657.

15. Tafa B, Sewunet T, Tassew H, Asrat D. Isolation and antimicrobial susceptibility patterns of Campylobacter species among diarrheic children at Jimma Ethiopia. Microbiol: Int J; 2014.

16. Brena MC, Mekonnen Y, Bettridge JM, Williams NJ, Wigley P, Tessema TS, Christley RM. Changing risk of environmental Campylobacter exposure with emerging poultry production systems in Ethiopia. Epidemiol Infect. 2016;144:567-75

17. Whiley H, van-den-Akker B, Giglio S, Bentham R. The Role of Environmental Reservoirs in Human Campylobacteriosis. Int J Environ Res. Public Health 2013, 10, 5886-5907.

18. Moher D, Liberati A, Tetzlaff J, Altman DG: Preferred reporting items for systematic reviews and meta-analyses: the PRISMA statement. BMJ 2009, 339.

19. Newcastle O: Newcastle- Ottawa: Scale customized for cross-sectional studies In. available from: https://static-contentspringer.com/esm//12889 _2012_5111_MOESM3_ESMdoc. Accessed 27 July 2018. In.; 2018.

20. Rücker G, Schwarzer G, Carpenter JR, Schumacher M: Undue reliance on 12 in assessing heterogeneity may mislead. BMC Med Res Methodol. 2008, 8(79).

21. Ioannidis JP. Interpretation of tests of heterogeneity and bias in metaanalysis. J Eval Clin Pract. 2008;14:1356-1294.

22. Lin L, Chu H. Quantifying Publication Bias in Meta-Analysis. Biometrics. 2018;74(3):785-94.

23. Borenstein M, Hedgesb LV, Higginsc JP, Rothsteind HR. A basic introduction to fixed-effect and random-effects models for meta-analysis. Res Syn Meth. 2010;1:97-111.

24. Beyene G, Haile-Amlak A. Antimicrobial sensitivity pattern of Campylobacter species among children in Jimma University Specialized Hospital Southwest Ethiopia. Ethio J Health Dev. 2004;18(3):185-9.

25. Kassa T, Gebre-Selassie S, Asrat D. Antimicrobial susceptibility patterns of thermotolerant Campylobacter strains isolated from food animals in Ethiopia. Vet Microbiol. 2007;119:82-7.

26. Woldemariam T, Asrat D, Zewde G. Prevalence of Thermophilic Campylobacter species in carcasses from sheep and goats in an abattoir in Debre Zeit area Ethiopia. Ethiop J Health Dev. 2009;23(3):229-33. 
27. Chanyalew Y, Asrat D, Amavisit P, Loongyai W. Prevalence and Antimicrobial Susceptibility of Thermophilic Campylobacter Isolated from Sheep at Debre Birhan, North-Shoa Ethiopia. Kasetsart J Nat Sci. 2013;47(4):551-60.

28. Nigatu S, Mequanent A, Tesfaye R, Garedew L. Prevalence and Drug Sensitivity Pattern of Campylobacter jejuni Isolated from Cattle and Poultry in and Around Gondar Town Ethiopia. Glob Vet. 2015;14(1):43-7.

29. Kebede A, Aragie S, Shimelis T. The common enteric bacterial pathogens and their antimicrobial susceptibility pattern among HIV-infected individuals attending the antiretroviral therapy clinic of Hawassa university hospital, southern Ethiopia. Antimicrob Resist Infect Control. 2017;6(128):1-7.

30. Getamesay M, Getenet B, Ahmed Z. Revalence of Shigella, Salmonella and Cmpylobacter Species and Their Susceptibility Patters among Under Five Children with Diarrhea in Hawassa Town South Ethiopia. Ethiop J Health Sci. 2014;24(2):1010-108.

31. Abamecha A, Assebe G, Belay T, Beyene W. Prevalence of Thermophilic Campylobacter and their Antimicrobial Resistance Profile in Food Animals in Lare District, Nuer Zone, Gambella Ethiopia. J Drug Res Dev. 2015;1(2):1-6.

32. Richardsona M, Garnera P, Doneganb S. Interpretation of subgroup analyses in systematic reviews: A tutorial. CEGH. 2019;7:192-8.

33. Igwaran A, Okoh Al. Human campylobacteriosis: A public health concern of global importance. Heliyon. 2019;5:e02814.

34. Costa D, Iraola G. Pathogenomics of Emerging Campylobacter Species. Clin Microbiol Rev. 2019;32(4):e00072-00018.

35. Sahin O, Yaeger M, Wu Z, Zhang Q: Campylobacter-Associated Diseases in Animals. Annu Rev Anim Biosci 2017, 12(26):9.1-9.22.

36. Gahamanyi N, Mboera LE, Matee MI, Mutangana D, Komba EV: Prevalence, Risk Factors, and Antimicrobial Resistance Profiles of Thermophilic Campylobacter Species in Humans and Animals in Sub-Saharan Africa: A Systematic Review. Int. J. Microbiol. 2020
37. Thomasa KM, de Glanvillec TW, Barkerd GC, Benschope J, Buzaf JJ, Cleaveland S. Prevalence of Campylobacter and Salmonella in African food animals and meat: A systematic review and meta-analysis. Int J Food Microbiol. 2020;315:108382

38. Terefe Y, Deblais L, Ghanem M, Helmy YA, Mummed B, Chen D. Cooccurrence of Campylobacter Species in Children From Eastern Ethiopia, and their association with environmental enteric dysfunction, diarrhea, and host microbiome. Front Public Health. 2020;8(9):1-16.

39. Silva J, Leite D, Fernandes M, Mena C, Gibbs PA, Teixeira P: Campylobacter spp. as a foodborne pathogen: a review. Front. Microbiol. 2011 2(200):1-12

40. Pallavi, Kumar A: Prevalence and antibiotic resistance pattern of Campylobacter species in foods of animal origin. Vet. World 2014, 7(9):681-684.

41. Sproston EL, Wimalarathna HM, Sheppard SK. Trends in fluoroquinolone resistance in Campylobacter. Microb. Genom. 2018;4:1-8.

42. Yang Y, Feye KM, Shi Z, Pavlidis HO, Kogut M, Ashworth AJ, Ricke SC.A Historical Review on Antibiotic Resistance of Foodborne Campylobacter. Front. Microbiol. 10:1509:.

43. Abdi RD, Mengstie F, Beyi AF, Beyene T, Waktole H, Mammo B, Ayana D, Abunna F. Determination of the sources and antimicrobial resistance patterns of Salmonella isolated from the poultry industry in Southern Ethiopia. BMC Infect Dis. 2017;17:352.

44. Gemeda BA, Amenu K, Magnusson U, Dohoo I, Hallenberg GS, Alemayehu G, Desta H, Wieland B. Antimicrobial Use in Extensive Smallholder Livestock Farming Systems in Ethiopia: Knowledge, Attitudes, and Practices of Livestock Keepers. Front. Vet. Sci. 2020; 7 (55):.

\section{Publisher's Note}

Springer Nature remains neutral with regard to jurisdictional claims in published maps and institutional affiliations.
Ready to submit your research? Choose BMC and benefit from:

- fast, convenient online submission

- thorough peer review by experienced researchers in your field

- rapid publication on acceptance

- support for research data, including large and complex data types

- gold Open Access which fosters wider collaboration and increased citations

- maximum visibility for your research: over $100 \mathrm{M}$ website views per year

At BMC, research is always in progress.

Learn more biomedcentral.com/submissions 